\title{
Determinations of ionosphere and plasmasphere electron content for an African chain of GPS stations
}

\author{
Andrew J. Mazzella Jr. ${ }^{1}$, John Bosco Habarulema ${ }^{2}$, and Endawoke Yizengaw ${ }^{3}$ \\ ${ }^{1}$ Watertown, Massachusetts, 02472-1049, USA \\ ${ }^{2}$ SANSA Space Science, Hermanus, 7200, South Africa \\ ${ }^{3}$ Institute for Scientific Research, Boston College, Chestnut Hill, Massachusetts, 02459, USA \\ Correspondence to: Andrew J. Mazzella Jr. (ajmazzella@brandeis.edu)
}

Received: 24 August 2016 - Revised: 15 March 2017 - Accepted: 27 March 2017 - Published: 3 May 2017

\begin{abstract}
The confluence of recent instrumentation deployments in Africa with developments for the determination of plasmasphere electron content using Global Positioning System (GPS) receivers has provided new opportunities for investigations in that region. This investigation, using a selected chain of GPS stations, extends the method (SCORPION) previously applied to a chain of GPS stations in North America in order to separate the ionosphere and plasmasphere contributions to the total electron content (TEC) during a day (24 July) in 2011. The results span latitudes from the southern tip of Africa, across the Equator, to the southern Arabian Peninsula, providing a continuous latitudinal profile for both the ionosphere and plasmasphere during this day.

The peak diurnal vertical ionosphere electron content (IEC) increases from about 14 TEC units (1 TEC unit $=10^{16}$ electrons $\mathrm{m}^{-2}$ ) at the southernmost station to about 32 TEC units near the geographic equator, then decreases to about 28 TEC units at the Arabian Peninsula. The peak diurnal slant plasmasphere electron content (PEC) varies between about 4 and 7 TEC units among the stations, with a local latitudinal profile that is significantly influenced by the viewing geometry at the station location, relative to the magnetic field configuration. In contrast, the peak vertical PEC varies between about 1 and 6 TEC units among the stations, with a more uniform latitudinal variation.

Comparisons to other GPS data analyses are also presented for TEC, indicating the influence of the PEC on the determination of latitudinal TEC variations and also on the absolute TEC levels, by inducing an overestimate of the receiver bias. The derived TEC latitudinal profiles, in comparison to global map profiles, tend to differ from the map results only about as much as the map results differ among themselves.
\end{abstract}

A combination of ionosonde IEC and alternative GPS TEC measurements, which in principle permits a PEC determination through their difference, was compared to the composite and separate ionosphere and plasmasphere contributions derived solely by the SCORPION method for one station. Although there is considerably more scatter in the PEC values derived from the difference of the GPS TEC and ionosonde IEC measurements compared to the PEC values derived by the SCORPION method, the average overhead values for this day are comparable for the two methods, near 2 TEC units, at the South African site examined.

This initial investigation provides a basis for day-to-day TEC monitoring for Africa, with separate ionosphere and plasmasphere electron content determinations.

Keywords. Ionosphere (equatorial ionosphere; instruments and techniques) - magnetospheric physics (plasmasphere)

\section{Introduction}

The SCORPION technique for calibration of Global Positioning System (GPS) total electron content (TEC) measurements was developed primarily to address the detrimental effects of the plasmasphere electron content (PEC) on the previous SCORE calibration technique (Lunt et al., 1999b), necessitating the development of a parametric representation for the plasmasphere as part of the calibration process (Mazzella et al., 2002, 2007). Previous studies included validations of the SCORPION technique for model data and corroborations against other methods for actual TEC measurements (Mazzella et al., 2007), and a more recent study demonstrated a method for resolving the remaining ambigu- 


\section{Africa GPS sites}

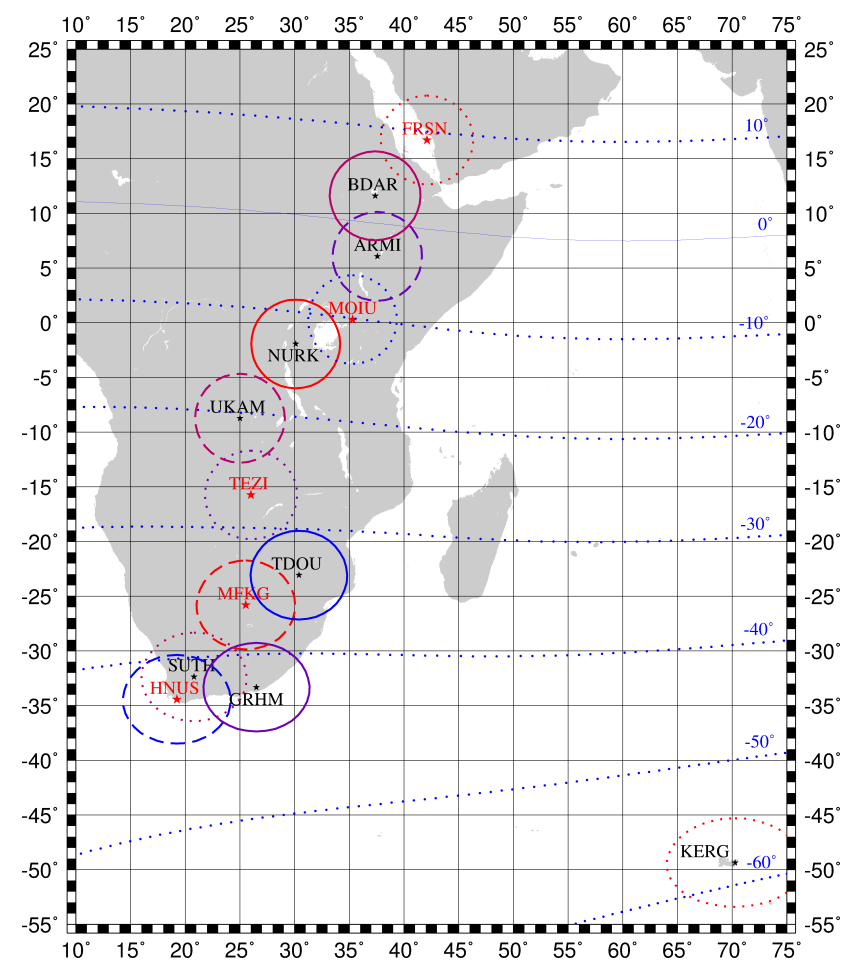

Figure 1. The GPS stations used for the Africa TEC profile for day 205, 2011, with their associated ionosphere coverage regions (ovals), corresponding to an elevation threshold of $35^{\circ}$ and an ionosphere altitude of $350 \mathrm{~km}$. Geomagnetic latitudes are shown in blue, and stations for which SUPIM values were calculated are shown in red text.

ity between the GPS receiver bias and an omnipresent uniform PEC contribution (Mazzella, 2012). That method requires a contiguous latitudinal chain of GPS stations, permitting the alignment of vertical PEC between pairs of stations to determine the locally omnipresent uniform PEC contribution (the "plasmasphere baseline").

Deployments of GPS receivers (and other ionospheric measurement systems) in Africa have significantly increased since 2008 (Yizengaw et al., 2013), permitting application of the same SCORPION analysis for a chain of stations on that continent. As with the previous North America study (Mazzella, 2012), a strict longitudinal alignment of stations is not required, because of the reliance on the local time dependence for the variations of the ionosphere electron content (IEC) and PEC, but a reasonable bound is required for the longitudinal differences between stations to minimize the effects of intrinsic variations for the ionosphere and plasmasphere. An initial bound of about $5^{\circ}$ in longitude was used for the selection of GPS stations, and the adequacy of this bound was confirmed by comparing the temporal TEC variations between neighboring stations, as well as between Hermanus, South Africa, and Grahamstown, South Africa.
Ionosphere reference altitudes

o Slant factor altitude

+ IPP altitude

Magnetic latitude

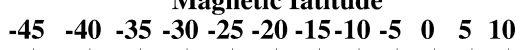

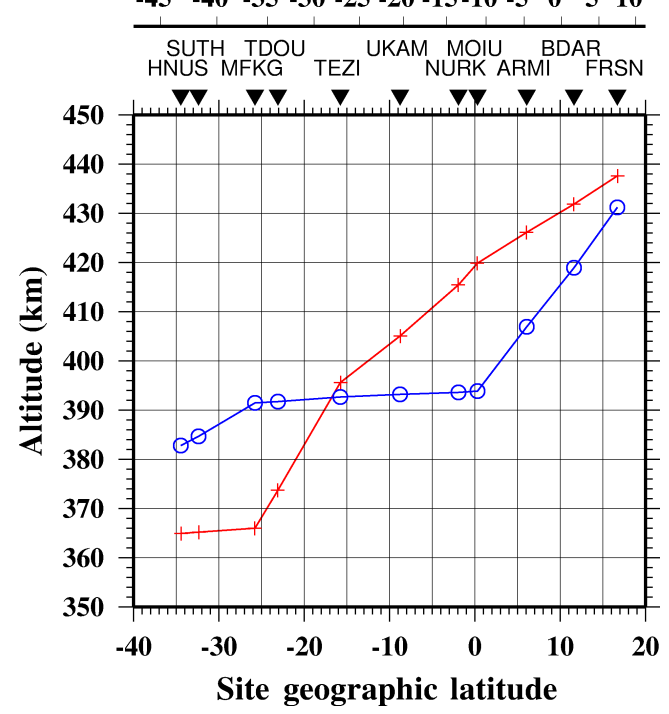

Figure 2. Ionosphere reference altitudes, used for the determination of the ionospheric penetration point (IPP) and the slant-to-vertical conversion for TEC, for each of the stations in the chain.

\subsection{Station and date selection}

An initial selection of GPS stations at strategic locations along the prospective latitudinal chain was examined for general data quality, including continuity and multipath. Replacement and supplementary stations were included to establish a complete chain with overlapping coverage for the ionosphere, using a $35^{\circ}$ elevation threshold, to restrict the errors imposed by the slant-to-vertical TEC conversions for the ionosphere (Andreasen et al., 1998). The time period for the study was chosen to be from the year 2011, during the early rising phase of the current solar cycle, for enhanced opportunities for quiet periods, which not only provide circumstances for more reliable calibrations but also allow extended periods for replenishment of the plasmasphere (Lunt et al., 1999a). Further subsets of this time period were then considered, based on the occurrence of active magnetospheric events (storms) that would disrupt and deplete the plasmasphere (and thus negate any meaningful determination of plasmasphere electron content), and additional accommodations were made based on data availability from individual stations, with some reconsideration of further alternatives for station selections. Ultimately, the data availability and geophysical activity determined a time period that was bounded by 7 July and 4 August 2011 .

The final selection of GPS stations used for this study is displayed in Fig. 1 and listed in Table 1 with their network associations. The ovals associated with each station indicate 
Table 1. The sites used for the Africa chain study, from south to north, with their supporting networks, and their derived plasmasphere baseline values (in TEC units).

\begin{tabular}{lrrllll}
\hline Long $(+\mathrm{E})$ & Lat $(+\mathrm{N})$ & MLat $(+\mathrm{N})$ & ID & Network & Site name & Baseline \\
\hline 70.256 & -49.351 & -58.280 & KERG & IGS & Kerguelen Islands & \\
19.223 & -34.424 & -42.663 & HNUS & IGS & Hermanus, South Africa & 0.000 \\
26.507 & -33.320 & -42.259 & GRHM & TRIGNET & Grahamstown, South Africa & \\
20.810 & -32.380 & -41.324 & SUTH & IGS & Sutherland, South Africa & 0.000 \\
25.540 & -25.805 & -36.270 & MFKG & IGS & Mafikeng, South Africa & 0.066 \\
30.384 & -23.079 & -33.893 & TDOU & IGS & Thohoyandou, South Africa & 0.082 \\
26.016 & -15.746 & -27.232 & TEZI & UNAVCO & Itezi-Tezi, Zambia & 0.294 \\
25.003 & -8.733 & -20.522 & UKAM & UNAVCO & Kamina, DR Congo & 0.370 \\
30.090 & -1.945 & -13.240 & NURK & IGS & Kigali, Rwanda & 0.922 \\
35.290 & 0.288 & -10.124 & MOIU & UNAVCO & Eldoret, Kenya & 1.189 \\
37.561 & 6.062 & -3.398 & ARMI & UNAVCO & Arba Minch University, Ethiopia & 1.905 \\
37.360 & 11.600 & 2.819 & BDAR & UNAVCO & Bahir Dar, Ethiopia & 1.913 \\
42.102 & 16.699 & 9.130 & FRSN & UNAVCO & Farasan, Saudi Arabia & 1.974 \\
\hline
\end{tabular}

the regional coverage, for an elevation threshold of $35^{\circ}$ and an ionosphere altitude of $350 \mathrm{~km}$. The station KERG was included to assess the higher-latitude plasmasphere content (primarily for the plasmasphere baseline evaluation), while the station GRHM was added for proximity to an ionosonde.

Prior to any extensive processing, an evaluation was conducted for two parameters that have a significant role for the calibration process. These parameters are the effective altitude for the (thin-layer) ionosphere, used for the calculation of the ionospheric penetration point (IPP) coordinates, and the slant factor altitude, used for the conversion from slant IEC to equivalent vertical IEC. As noted by Mazzella (2009), these two altitude quantities need not be equal. The evaluation of the two altitude quantities was conducted using the Sheffield University Plasmasphere Ionosphere Model (SUPIM; Bailey and Balan, 1996) for five of the stations in the chain (HNUS, MFKG, TEZI, MOIU, and FRSN) for geophysical parameters typical of the conditions during the period selected $(\mathrm{F} 10.7=125, \mathrm{~F} 10.7 \mathrm{av}=98.6, \mathrm{Ap}=27)$. The procedure for this evaluation is presented in the Appendix. The results for all stations are displayed in Fig. 2, using linear interpolation for station locations where SUPIM calculations were not performed. For the slant factor altitude, interpolation was also performed for TEZI, because anomalous results for that quantity were derived using SUPIM directly.

A notable feature in Fig. 2 is that the IPP altitude becomes greater than the slant factor altitude for TEZI and equatorward stations. This occurrence is attributed to the local TEC gradients and the limitations of the method for the altitude determinations, but contradicts the conjecture by Mazzella (2009) that the difference between the slant factor altitude and the IPP altitude can be interpreted as an effective thickness for the ionosphere.

The GPS receiver at Hermanus, South Africa (HNUS), was used for a survey of the ionosphere and plasmasphere conditions for the entire period from 7 July to 4 August 2011, except for 20 July 2011, for which data were unavailable. The most significant PEC occurred for 23 July 2011 (day 204), but examination of other stations indicated active conditions for the ionosphere, reducing both the accuracy of the calibrations and the partitioning of TEC between the ionosphere and plasmasphere. Consequently, the data were analyzed for 24 July 2011 (day 205), for which the PEC values were only slightly lower and quiet ionospheric conditions $(\mathrm{Ap}=4, \Sigma \mathrm{Kp}=9,-9 \leq \mathrm{Dst} \leq 0)$ prevailed for all stations.

\subsection{Data processing}

The initial processing of the RINEX (Receiver INdependent EXchange format) data files for each station was performed using the GPS Toolkit developed by the Applied Research Laboratory (ARL) of the University of Texas (Tolman et al., 2004), using data for two contiguous days to obtain complete satellite passes spanning a period of more than $24 \mathrm{~h}$ (Mazzella, 2012). Further examination and editing were conducted for each satellite pass to eliminate outliers for dispersive carrier phase and dispersive group delay, remove any residual phase discontinuities, and eliminate data segments affected by missing ephemeris data. The standard practice of aligning dispersive carrier phase to dispersive group delay was performed (e.g., Ma and Maruyama, 2003), without elevation-dependent weighting, followed by additional corrections for multipath to minimize bias discrepancies for different passes by the same satellite (Kee and Parkinson, 1994; Andreasen et al., 2002).

All GPS stations were initially calibrated by SCORPION individually, but preliminary inter-comparisons for adjacent stations indicated discrepancies of up to 5 TEC units (1 TEC unit $=10^{16}$ electrons $\mathrm{m}^{-2}$ ) for the ionosphere diurnal profile levels between some stations. Consequently, to improve the correspondence for the ionosphere TEC profiles and the associated partitioning of TEC into ionosphere and plasmas- 

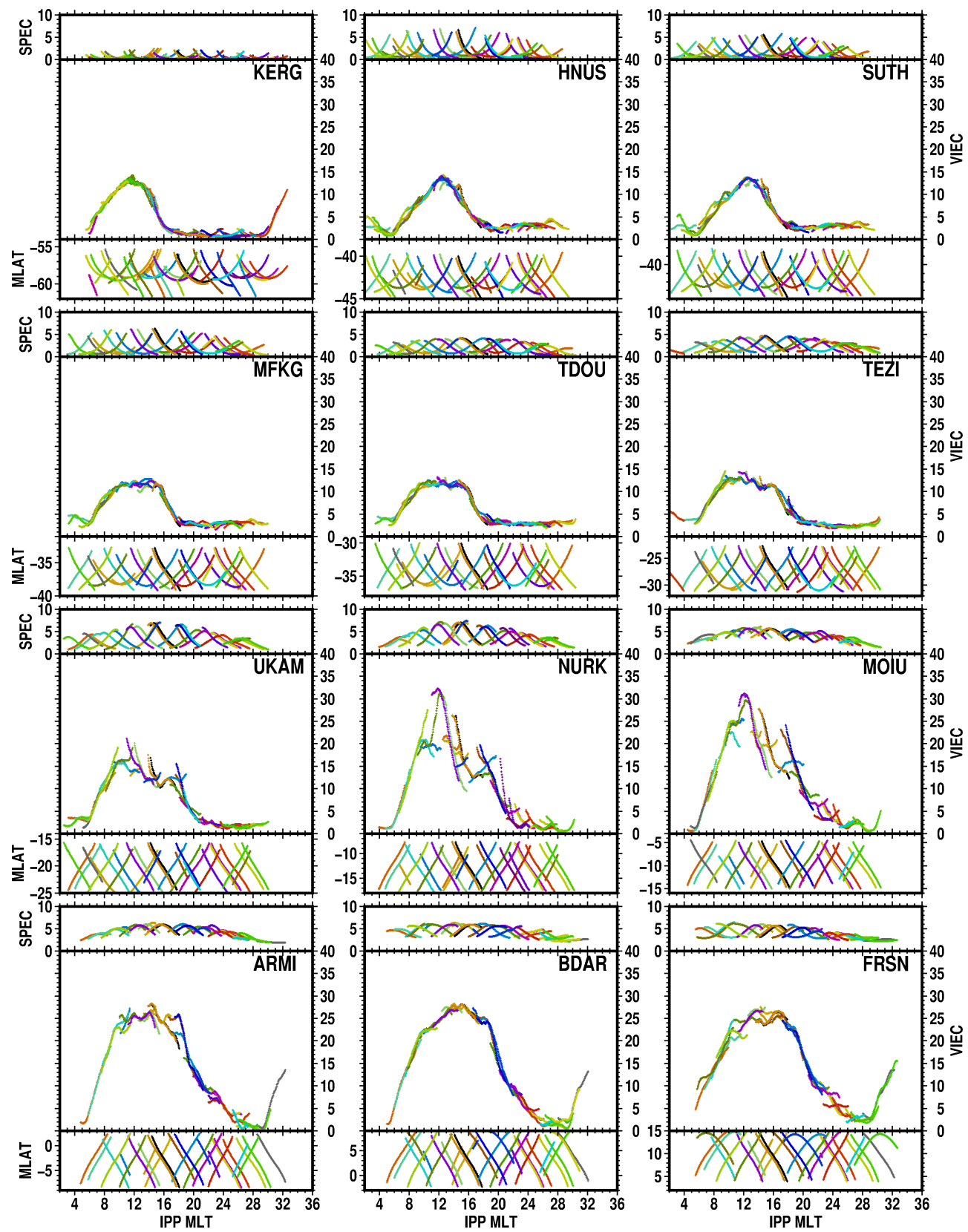

Figure 3. The diurnal TEC variations for the GPS stations, displaying, for each station, the magnetic latitude of the IPP in the bottom panel, the equivalent vertical ionospheric electron content (VIEC) in the middle panel, and the slant plasmaspheric electron content (SPEC) in the top panel, versus the magnetic local time at the IPP. Data segments are color-coded by GPS satellite.

phere components, "referenced calibrations" (Andreasen et al., 1998) were performed for stations from MFKG to FRSN. In each case, the neighboring southward station was used for the known reference TEC values to assist in the calibration of the northward station of the pair, so the calibrations proceeded from south to north along the chain. For most of the chain, this corresponded to an equatorward progression, initiated from the poleward station with the lowest plasmasphere contribution.

\section{Analysis results}

\subsection{Inter-station comparisons}

The TEC determinations for all of the stations are presented in Fig. 3, displaying, from bottom to top panels, the magnetic latitude (MLAT) of the IPP, the equivalent vertical ionospheric electron content (VIEC), and the slant plasmaspheric electron content (SPEC) against the magnetic local time (MLT) at the IPP. As in the previous studies by 
Mazzella $(2009,2012)$, the boundary between the ionosphere and plasmasphere is considered to be at an altitude of $1000 \mathrm{~km}$, for all latitudes, and is thus distinct from a boundary based on geophysical quantities. The peak VIEC increases significantly between TDOU and NURK, by more than a factor of 2, while the SPEC also increases somewhat. The latitudinal variations of SPEC (Fig. 3), both for each GPS station and across all stations, display characteristics that overlap with the previous North America study (Mazzella, 2012), but are distinctly different in the equatorial region. The smoothness of the PEC results is consistent with the large volume and diffuse density distribution of the plasmasphere.

The TEC comparisons for overlapping magnetic latitude bands, used to establish the plasmasphere baseline for each station except HNUS, are displayed in Fig. 4. Here, the vertical plasmaspheric electron content (VPEC) is displayed in the top section of each panel for each station. This quantity is not directly measured, but is determined by the parametric representation for the plasmasphere, as derived by SCORPION.

The correspondence for VIEC values generally reflects the usage of the referenced calibration method, but the comparisons between HNUS and SUTH are for independently calibrated stations. The plasmasphere baseline values, derived progressively beginning from HNUS, have been applied prior to generating this display, and eliminated any relative displacements of the paired VPEC profiles. These plasmasphere baseline values are presented in Table 1. The assignment of zero for the plasmasphere baseline for HNUS was confirmed by extrapolation from KERG, but is also consistent with the zero plasmasphere baseline determined for stations at similar magnetic latitudes for the North American chain (Mazzella, 2012).

A further observation from Fig. 4 arises from the occurrence of vertical TEC (VTEC) profiles for southern and northern regions relative to an individual station in successive plots, indicating the transformation of the diurnal profile with latitude. The most significant change occurs for NURK, but UKAM and MOIU also display significant changes. These TEC changes with latitude are directly displayed in Fig. 5, for four magnetic local times (06:00, 12:00, 18:00, and 24:00, clockwise from upper left). For each local time, the VIEC appears in the bottom panel, the VPEC appears in the top panel, and their sum, the composite VTEC, appears in the center panel. Also displayed in these figures are IONEX TEC results from the Center for Orbit Determination in Europe (CODE) of the Astronomical Institute of the University of Bern, the European Space Agency (ESA), the Jet Propulsion Laboratory (JPL) of the California Institute of Technology, and the Research Group of Astronomy and Geomatics of the Technical University of Catalonia (UPC) analysis centers. The IONEX TEC results correspond to the composite VTEC, but are also displayed with the vertical ionosphere TEC for comparison.

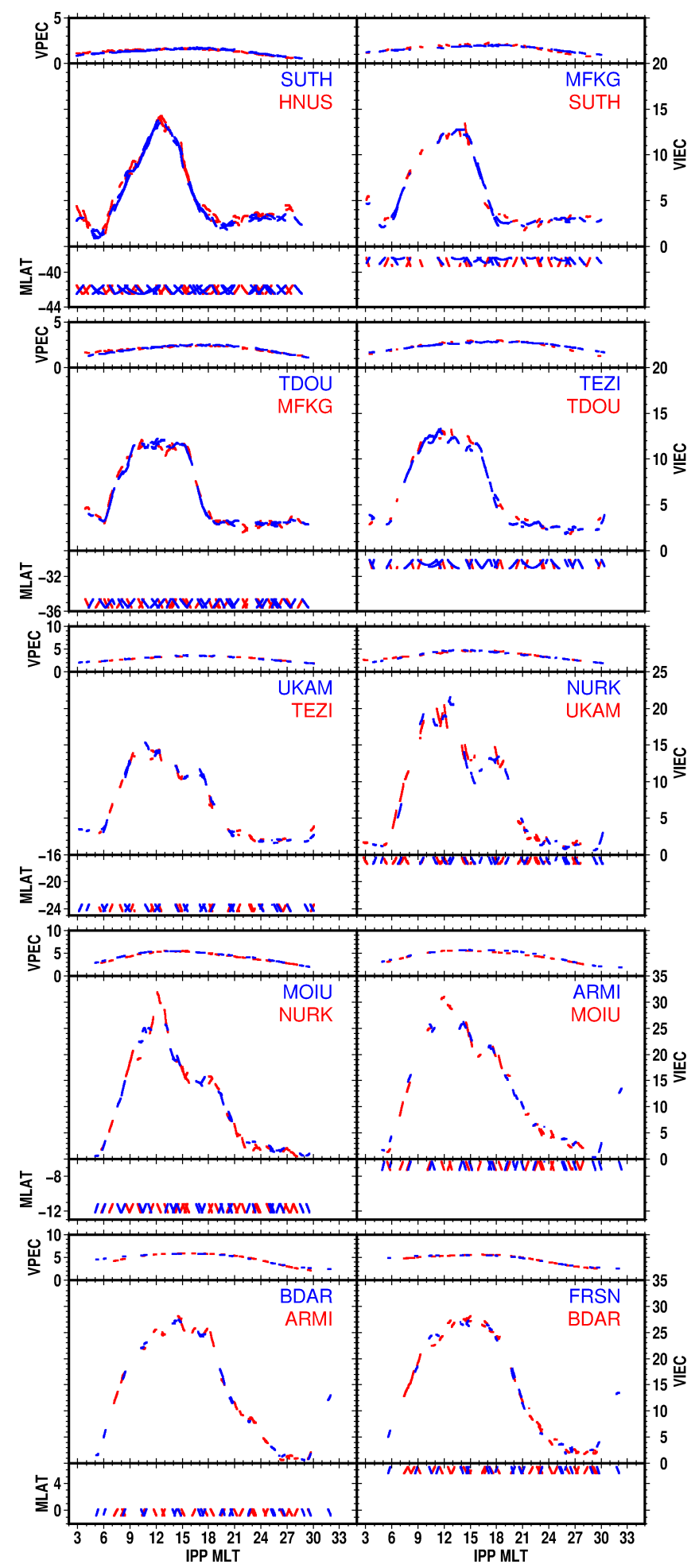

Figure 4. The TEC comparisons for overlapping $1^{\circ}$ magnetic latitude bands, for the determination of the plasmasphere baselines. The vertical plasmaspheric electron content (VPEC) is displayed in the top of each panel for each station. 

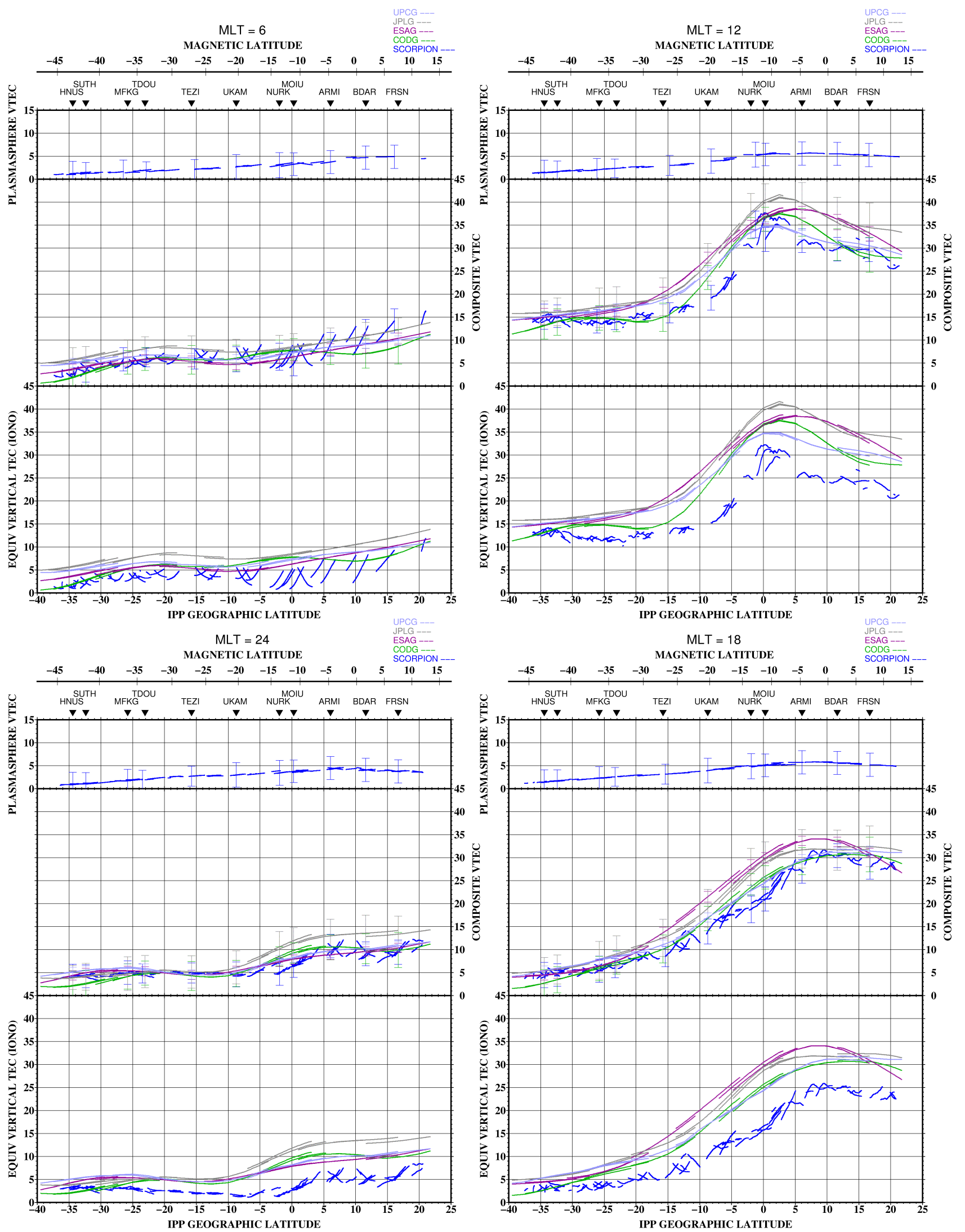

Figure 5. Latitudinal vertical TEC profiles for the separate ionosphere (bottom panel) and plasmasphere (top panel), with the composite vertical TEC (middle panel), for the SCORPION results and the CODE, ESA, JPL, and UPC IONEX maps, for magnetic local times (clockwise from upper left) 06:00, 12:00, 18:00, and 24:00. 
Table 2. Receiver biases and error estimates, in TEC units, from SCORPION, IONEX maps, and the July 2011 CODE monthly report, indicating whether the coarse acquisition $(\mathrm{C} 1)$ or precise code $(\mathrm{P} 1)$ signal is used for the L1 frequency.

\begin{tabular}{llrl|rr|rr|rr|rr|rr}
\hline Site & Bias & \multicolumn{2}{c}{ SCORPION } & \multicolumn{2}{c|}{ CODG } & \multicolumn{2}{|c|}{ ESAG } & \multicolumn{2}{|c|}{ JPLG } & \multicolumn{2}{c}{ CODE (2011-07) } \\
\hline ID & Type & Bias & Error & Bias & Error & Bias & Error & Bias & Error & Bias & Error \\
\hline KERG & P1-P2 & 9.189 & 1.629 & 9.231 & 0.200 & 8.800 & 0.400 & 6.968 & 0.091 & 12.425 & 1.877 \\
HNUS & C1-P2 & 57.714 & 2.736 & & & & & & & & \\
SUTH & P1-P2 & 21.078 & 2.457 & 23.656 & 0.126 & 20.033 & 0.400 & 23.407 & 0.080 & 23.293 & 0.129 \\
MFKG & C1-P2 & -42.821 & 2.545 & & & & & & & & \\
TDOU & C1-P2 & -58.940 & 2.083 & & & & & & & & \\
TEZI & C1-P2 & 44.638 & 2.207 & & & & & & & & \\
UKAM & C1-P2 & -46.873 & 2.717 & & & & & & & & \\
NURK & P1-P2 & 50.606 & 2.924 & 46.132 & 0.120 & 81.664 & 1.291 & & & 46.083 & 0.257 \\
MOIU & P1-P2 & 2.429 & 2.624 & & & & & & & & \\
ARMI & C1-P2 & -44.308 & 2.578 & & & & & & & & \\
BDAR & C1-P2 & -36.798 & 2.576 & & & & & & & & \\
FRSN & C1-P2 & -36.970 & 2.620 & & & & & & & & \\
\hline
\end{tabular}

In Fig. 5, the GPS data were selected over an interval of $\pm 0.5 \mathrm{~h}$ in magnetic local time relative to the nominal magnetic local time for each figure, using an elevation threshold of $35^{\circ}$, while the IONEX data were selected along the local geographic meridian for each station at the nominal magnetic local time. The $1 \mathrm{~h}$ selection interval for the GPS data allows some intrinsic temporal variation, accounting for the contravening latitudinal TEC gradients of some of the data segments, which is especially noticeable for MLT $=6$ and MLT $=18$. The SCORPION GPS data Plasmasphere VTEC errors are estimates, calculated as the square root of the peak plasmasphere slant TEC (STEC), while the Composite VTEC errors also include additional contributions arising from the discrepancies for local vertical Ionosphere TEC values at conjunctions. The CODE, ESA, JPL, and UPC error values were provided with the respective bias values. The magnetic latitudes indicated above the plot correspond to those along a magnetic longitude of $99.61^{\circ}$, which is the average magnetic longitude for the chain of stations.

A review of the IONEX GPS station locations indicated an absence of coverage for the African latitude band between about 10 and $15^{\circ} \mathrm{S}$ for all four IONEX analysis centers, so the associated VTEC values for this band result from the map interpolation. A similar circumstance applies only to the JPL IONEX map for the African latitude band between about 5 and $15^{\circ} \mathrm{N}$.

Based on both the SCORPION and IONEX results, the equatorial ionosphere anomaly is absent for this day over Africa, although the peak ionosphere VTEC does occur as far south as $10^{\circ} \mathrm{S}$ magnetic latitude (near the geographic equator) during the late morning (10:00-12:00 MLT), before migrating to the magnetic equator. Extended latitudinal coverage provided by the IONEX files indicates the absence of a corresponding local TEC maximum for northern magnetic latitudes during the morning hours.
For the nighttime case (MLT $=24$ ), the VPEC is comparable to the VIEC between TEZI and MOIU and is a significant fraction of the composite VTEC for all of the stations. This circumstance matches the nighttime midlatitude PEC report by Lunt et al. (1999a) based on SUPIM calculations and by Yizengaw et al. (2008) using JASON-1 PEC estimation, but the relative VPEC is larger than the nighttime VPEC / VIEC ratio (0.05-0.25) reported by Lee et al. (2013), also using JASON-1 measurements, for 2002-2009. There is also a small residual VIEC peak near the magnetic equator, but the latitudinal coverage for this set of stations does not allow for a determination of whether this is part of a higher VIEC region northward of the chain.

\subsection{Diurnal VTEC comparisons to other methods}

For a comparison of TEC results between SCORPION and a more conventional GPS TEC determination method, the Boston College Institute for Scientific Research (BC/ISR) calibration technique was chosen as an alternative (Valladares et al., 2009). For the latter technique, the uncalibrated dispersive, phase-aligned TEC measurements are corrected for the relative GPS satellite biases reported by the University of Bern (ftp://ftp.unibe.ch/aiub/CODE), and the receiver bias is determined by minimizing the equivalent VTEC variability between 02:00 and 06:00 local time (LT). The calibrated STEC values are converted to equivalent VTEC using a conversion altitude of $350 \mathrm{~km}$.

Four stations (HNUS, TEZI, MOIU, and FRSN) within the chain were selected for this comparison, and the quantities compared included the BC/ISR equivalent vertical TEC (VTEC) against the ionosphere equivalent vertical TEC (VIEC), the composite equivalent vertical TEC (CEQV), and the composite ionosphere and plasmasphere vertical TEC (CVTC) determined by SCORPION. Of the SCORPION quantities, the CEQV is closest in definition to the BC/ISR 


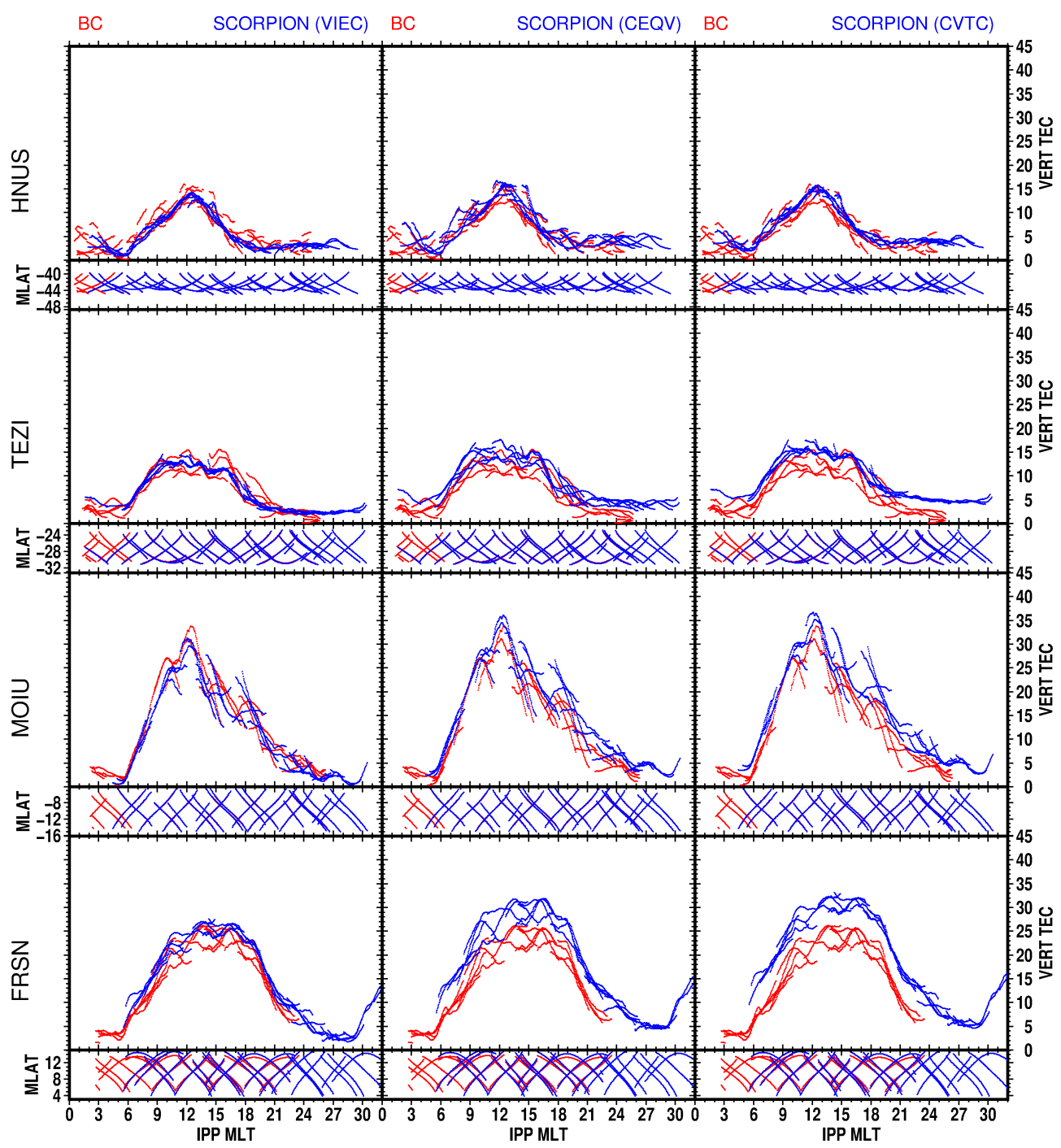

Figure 6. Overlays of SCORPION vertical TEC (blue) and BC/ISR vertical TEC (ionosphere and plasmasphere) (red), for SCORPION VIEC (ionosphere only) in the left column, CEQV (composite equivalent vertical ionosphere and plasmasphere) in the center column, and CVTC (composite vertical ionosphere and plasmasphere) in the right column.

VTEC, for the conversion of the combined slant plasmasphere and ionosphere TEC to equivalent vertical, but differs through the biases and altitudes associated with the IPP and slant factor definitions. The CVTC from SCORPION is the closest quantity to true VTEC, being calculated as the combination of the VIEC and the SCORPION representation for the local vertical PEC (VPEC) at the IPP. (The VIEC, CVTC, and VPEC were previously displayed in Fig. 5, across latitudes.)

For the comparison of the BC/ISR VTEC against the SCORPION VIEC (Fig. 6, left column), the VIEC diurnal profiles for HNUS, TEZI, and FRSN are distinctly smoother than the BC/ISR VTEC profiles, with a lesser difference for MOIU. This difference is attributable to the plasmasphere, as noted by Mazzella (2009), and is further supported by the comparison of the BC/ISR VTEC against the SCORPION
CEQV (Fig. 6, middle column), for which the variations at each local time are quite similar and the TEC differences are primarily for bias levels. A lesser variation for the SCORPION CVTC against the BC/ISR VTEC is evident (Fig. 6, right column), indicating the role of the IPP reference location for the plasmasphere vertical electron content determination in ameliorating the apparent gradients induced by converting between slant and vertical PEC using the same conversion as for the ionosphere.

An additional comparison for alternative measurements was provided by the ionosonde and GPS receiver (GRHM) at Grahamstown, South Africa (McKinnell et al., 2007). (Data from the ionosonde at Hermanus were unavailable for day 205,2011 .) The method utilized was previously described and implemented by Belehaki et al. (2003), in which a topside extension of ionosonde measurements to $1000 \mathrm{~km}$ alti- 


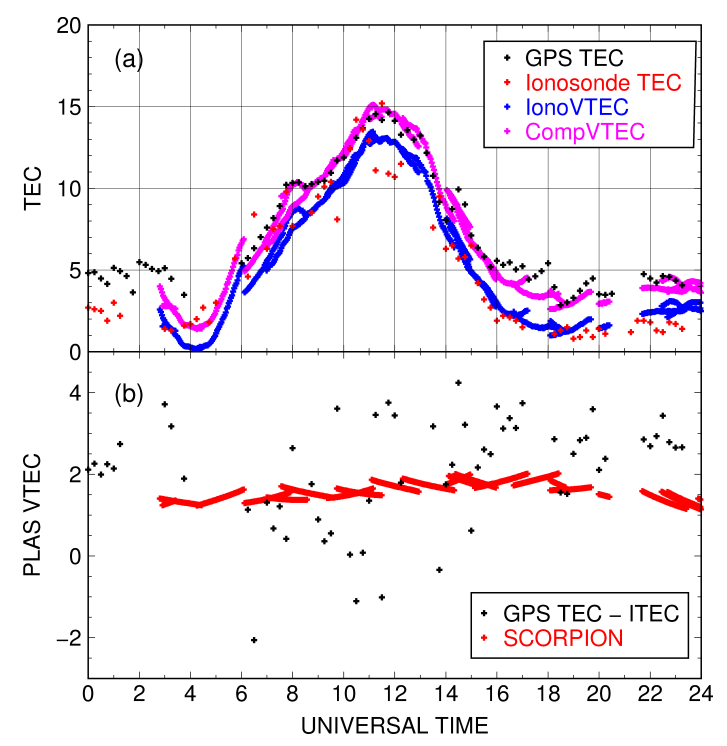

Figure 7. (a) Ionosonde vertical TEC (red), BC/ISR GPS vertical TEC (above $60^{\circ}$ elevation; black), SCORPION composite vertical TEC (above $60^{\circ}$ elevation; "CompVTEC", pink), and SCORPION ionosphere-only vertical TEC (above $60^{\circ}$ elevation; "IonoVTEC", blue); (b) BC/ISR GPS vertical TEC minus ionosonde vertical TEC (black) and SCORPION plasmasphere-only vertical TEC (red).

tude is used to determine the VIEC (Huang and Reinisch, 2001) while the GPS receiver provides the combined IEC plus PEC. The TEC data for GRHM were processed by SCORPION, and separately using the BC/ISR method, and the latter results were combined with the ionosphere TEC (ITEC) estimates from the ionosonde, after selecting GPS data for elevations at or above $60^{\circ}$ (Fig. 7). The BC/ISR VTEC ("GPS TEC") values generally lie near the SCORPION CVTC ("CompVTEC") values, and also generally above the ionosonde ITEC estimates, except for a few points, primarily around midday (Fig. 7a). The difference between the BC/ISR VTEC and the ionosonde ITEC was evaluated as a plasmasphere electron content measurement (PTEC) and compared to the VPEC from SCORPION (Fig. 7b). The scatter for the GPS TEC minus ITEC values is considerably larger than for the SCORPION VPEC, for which the primary variation is the latitudinal gradient over the $3^{\circ}$ spanned by the data.

The receiver biases for each station were calculated as the average bias over all satellites, and are listed in Table 2, with corresponding values (if available) from tabulations in the IONEX files or reported by CODE. The ESA IONEX receiver bias for NURK is significantly different from the SCORPION, CODE IONEX, and CODE monthly report biases, and would produce negative TEC values if applied to the measurements for that station. Similar receiver bias values are reported in the ESA IONEX files for adjacent days, so this does not appear to be an isolated anomalous value, but may be indicative of the plasmasphere influence on the
ESA calibration process. Such an overestimate of the biases for equatorial regions was noted by Mazzella et al. (2007), Anghel et al. (2009), and Carrano et al. (2009), for different calibration methods that did not incorporate determinations of the plasmasphere electron content.

The associated derived relative satellite biases, resulting from the removal of the receiver bias, are displayed in Fig. 8, separately for the receivers for which the coarse acquisition (C1) signal is used (Fig. 8a) instead of the precise code (P1) signal (Fig. 8b). For comparison, the relative satellite biases reported by CODE for July 2011 are also displayed. The agreement among the stations and with CODE is generally within 3 TEC units (with 1 TEC unit corresponding to $0.350 \mathrm{~ns}$ of differential delay), although there are several discrepant relative bias differences exceeding that bound, notably for NURK, a site for which the multipath was more significant than most of the other sites. The PRN 1 CODE P1-P2 bias (23.0503 TEC units) for July 2011 is distinctly different from the CODE value (30.6156 TEC units) reported for August 2011, possibly reflecting the limited time for evaluation after the launch of PRN 1 on 16 July 2011 (www.gpsworld.com/the-almanac/).

\section{Discussion and conclusions}

A quiet-day study for a latitudinal chain in Africa was conducted as an initial investigation for that region using the SCORPION method for partitioning the ionosphere and plasmasphere electron content contributions, both to improve the quality of the TEC calibrations and to evaluate more representative values for the VTEC. Standard procedures for converting STEC to equivalent VTEC utilize the combined slant IEC and PEC contributions along the line of sight. However, except for nearly vertical lines of sight, this PEC contribution is from a more distant region than the IPP, so the horizontal PEC gradient is compressed into a more steeply varying quantity by mapping this contribution to the IPP (Mazzella, 2009). This gradient enhancement appears as a "sawtooth" pattern of steeper, disjoint TEC gradients distinct from the general TEC trend across several sites (Mazzella, 2012). The absence of this sawtooth pattern in the composite VTEC latitudinal profiles (Fig. 5) is an indication of the quality of the current results. The smooth variation of the vertical PEC with latitude indicates the consistency of the regional plasmasphere determinations at each site, as enforced by both the referenced calibrations and the plasmasphere baseline matching process.

Although referenced calibrations were not required for the North America chain (Mazzella, 2012), and plasmasphere baseline adjustments were marginal for that investigation, both of those features of the SCORPION method had significant roles for the Africa chain, because of the nature of the ionosphere and plasmasphere at lower latitudes. Based on SUPIM studies, Mazzella (2009) indicated the possibil- 
(a) Relative satellite biases (C1 usage)

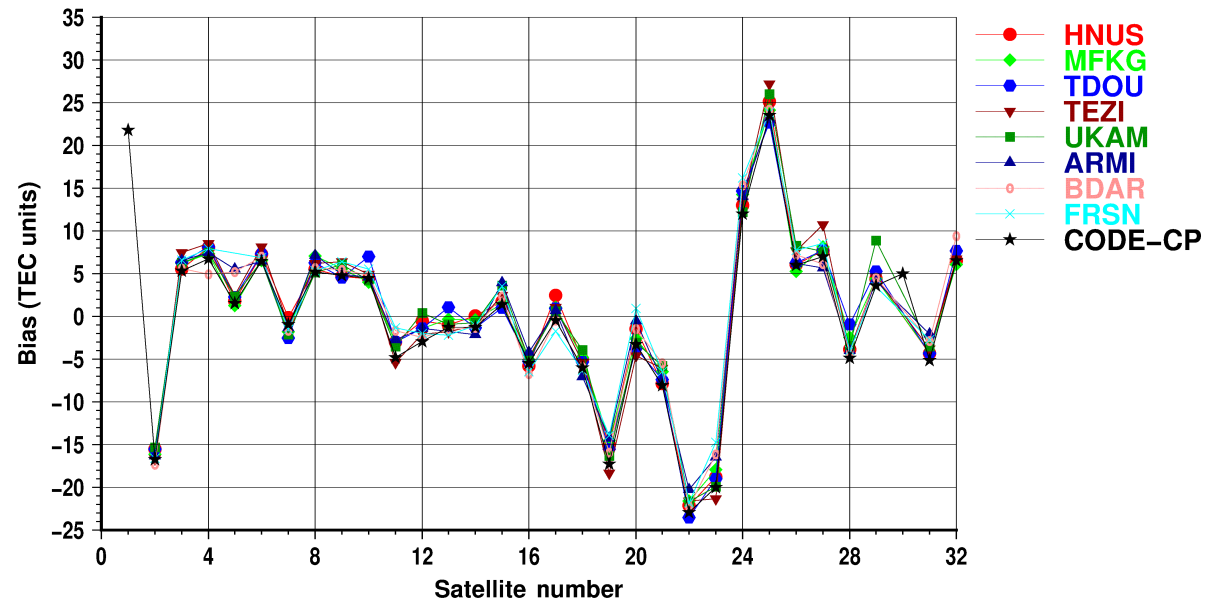

(b)

Relative satellite biases (P1 usage)

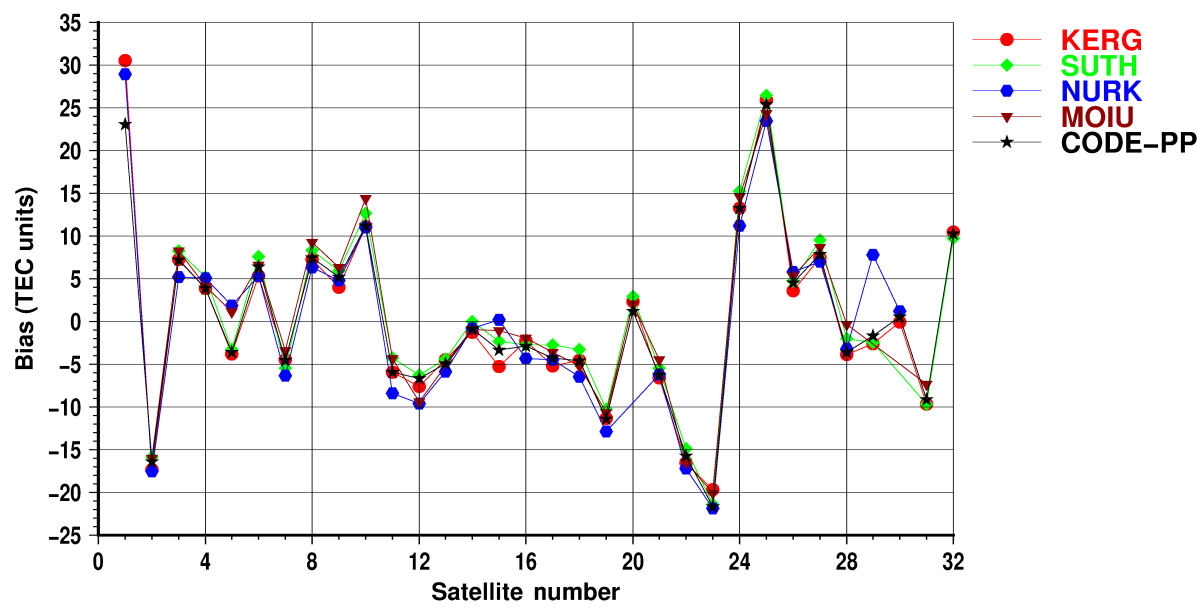

Figure 8. The derived relative satellite biases, resulting from the removal of the receiver bias, separately for the receivers for which the coarse acquisition (C1) signal is used (a) instead of the precise code (P1) signal (b).

ity of different effective altitudes for the ionospheric penetration point and slant factor conversion at different local times, but similar evaluations for lower latitudes (also using SUPIM) indicate the prospect of varying effective altitudes with azimuth for a single local time, even with the exclusion of the plasmasphere contribution. In combination with the tasks of disentangling the bias effects and plasmasphere contributions from the ionosphere electron content, the possible variation for the slant factor conversion is an additional complication, although the apparent resolution by means of the referenced calibrations is somewhat encouraging. A caveat regarding the results is the possible progressive distortion of the TEC profiles along the chain of sites, but the individual diurnal and latitudinal profiles appear to indicate relatively restrictive limits regarding this possible occurrence.

A further favorable result in this study was the avoidance of negative TEC excursions for the equatorial region, as a consequence of the influence on the bias determinations by the plasmasphere when it is not explicitly treated within the calibration process (Mazzella et al., 2002, 2007; Anghel et al., 2009; Carrano et al., 2009; Mazzella, 2012). Consequently, the associated latitudinal trend for the receiver bias error (Mazzella, 2012) has also been significantly reduced. Accommodating latitudinally varying plasmasphere content for the accuracy of the calibration process provides some encouragement that the day-to-day temporal variation of the plasmasphere content will also have less influence on the derived receiver biases, so that a possibly spurious variability of receiver bias values can be eliminated.

The prospect for day-to-day TEC monitoring, with ongoing bias determinations and partitioning between the ionosphere and plasmasphere, requires addressing the detrimental effects of ionospheric activity on the calibration process. Such ionosphere activity invalidates the assumption of equal equivalent vertical IEC values for "common" IPP observations (with the operational definition of "common" being implemented by the parameters defining conjunctions). Use of relative (satellite) biases within the calibration process re- 
duces the number of parameters to be determined by the calibration, allowing more restrictive conjunction limits for comparisons of equivalent vertical IEC values, especially for limiting universal time differences between conjunction pairs, thus reducing the time allowed for intrinsic ionospheric variations. For GPS stations that have previously been fully calibrated on a quiet day, as in this study, the set of relative satellite biases for the station may be more advantageous for use than a published globally derived set of relative satellite biases (such as those from CODE), because some effects ameliorating residual multipath will be advantageously incorporated. Using relative biases may also allow the activity occurrences within a calibration period to be excluded, while still allowing subsequent use of the TEC data for the active interval. However, further investigation and experiments are required for such an implementation, because of the uncertain effects on the partitioning between the ionosphere and plasmasphere.

Addressing the direct and indirect influences of the plasmasphere on TEC measurements for Africa is a significant development, for improving both the quality and the interpretation of the measurements. The influence of the plasmasphere content on the bias determinations and derived IEC latitudinal gradients has been noted previously (Mazzella et al., 2007; Mazzella, 2012), but the gradual replenishment and storm-related depletion of the plasmasphere produce circumstances for masquerading as seasonal or other medium-term effects, unless addressed by additional investigations, such as the superposed epoch analysis by Lunt et al. (1999c).

The current study included comparisons of SCORPION results against a more conventional single-station GPS TEC analysis method (BC/ISR), as well as against multi-station, global mapping TEC representations (IONEX) for four separate analysis centers (CODE, ESA, JPL, and UPC). The SCORPION comparisons to the BC/ISR results displayed the anticipated difference in VTEC levels between calibration methods with explicit or implicit treatments of the plasmasphere content, with the associated bias overestimate at lower latitudes when the plasmasphere content is not explicitly determined (Mazzella et al., 2007). The VTEC variations with latitude are also embellished when the plasmasphere content is not treated separately. The SCORPION CVTC latitudinal profiles, in comparison to the IONEX profiles, tend to differ from the IONEX results only about as much as the IONEX results differ among themselves, although the CODE and UPC results appear to match more closely with SCORPION, especially for magnetic local times between 12:00 and 16:00.
The ionosonde-with-GPS method (Belehaki et al., 2003) provides an alternative ground-based technique for separate ionosphere and plasmasphere electron content determinations, but requires both an accurate scale height determination from the ionosonde measurements and an accurate calibration for the GPS TEC in the presence of plasmasphere content. The large (5 TEC units) and irregular variations in the plasmasphere electron content derived by this method (Fig. 7b) indicate difficulties with either or both of those instrumental parameter determinations. The previous discussion concerning the GPS TEC determinations would indicate that the problem is not solely with that measurement. The discrepancies between the ionosonde TEC and GPS TEC noted by Fremouw et al. (2001) and Secan et al. (2005) for higher northern latitudes, where the plasmasphere contribution is insignificant, indicate considerable uncertainty for the ionosonde TEC determinations.

A remaining data source for PEC comparison data is the set of measurements from the GPS receiver aboard the JASON-2 satellite. (Comparisons of IEC, using the JASON2 altimeter data, are considerably restricted for the chain of stations used in the current study, because of their inland locations.) A supplementary analysis similar to that by Yizengaw et al. (2008) and Lee et al. (2013) is being conducted specifically for Africa on the day of this case study to evaluate PEC values for comparison to the SCORPION results.

Data availability. Data associated with Figs. 2-8 are provided as text tabulations in the Supplement. 


\section{Appendix A: Calculation of reference ionosphere altitudes}

i. Altitude for IPP location (HgtIPP):

a. Generate azimuth slant TEC (STEC) scans from a model, at $2 \mathrm{~h}$ intervals, for a $35^{\circ}$ elevation and azimuths from 0 to $360^{\circ}$ at $10^{\circ}$ intervals. (Because HgtIPP is an ionosphere reference altitude, an upper limit of $1000 \mathrm{~km}$ altitude is used for the STEC calculations, consistent with the nominal ionosphere/plasmasphere boundary altitude noted in Sect. 2.1.)

b. Determine the median altitudes, using the cumulative STEC altitude profile for the azimuth scans.

c. For each $2 \mathrm{~h}$ interval, calculate an IPP altitude as an STEC-weighted average of the median altitudes over all azimuths.

d. Calculate the representative IPP altitude (HgtIPP) as the maximum of the individual azimuthal STECweighted average IPP altitudes. (For calibrations, this was considered to be the most appropriate choice, compared to a median or average value.)

ii. Altitude for slant factor (AltIPP):

a. Generate meridian STEC scans from a model, for elevations from 0 to $90^{\circ}$ at $5^{\circ}$ increments, for azimuths 0 and $180^{\circ}$, at $2 \mathrm{~h}$ intervals. (These STEC values are also calculated using an upper altitude limit of $1000 \mathrm{~km}$.)

b. Generate latitudinal true VTEC profiles from the model, for latitudes between -55 and $55^{\circ}$ at $2^{\circ}$ increments, at $2 \mathrm{~h}$ intervals. (Likewise, these VTEC values are calculated using an upper altitude limit of $1000 \mathrm{~km}$.) c. For elevations above a threshold $\left(35^{\circ}\right)$, minimize the following quantity for each $2 \mathrm{~h}$ interval,

$$
\begin{aligned}
E & =\sum_{i}\left(\operatorname{VTEC}_{i}\right. \\
& \cdot \sec \left(\arcsin \left(\left(\frac{R_{\mathrm{e}}}{R_{\mathrm{e}}+\operatorname{AltIPP}}\right) \cos \left(\varepsilon_{i}\right)\right)\right) \\
& \left.-\mathrm{STEC}_{i}\right)^{2},
\end{aligned}
$$

to solve for the slant factor altitudes (AltIPP) for every $2 \mathrm{~h}$, for $R_{\mathrm{e}}=$ Earth's radius and $\varepsilon_{i}=$ elevation. The $\mathrm{VTEC}_{i}$ value corresponding to $\mathrm{STEC}_{i}$ is determined by interpolation from the true VTEC latitudinal profile to the IPP latitude associated with sample $\mathrm{STEC}_{i}$. (Thus, the IPP reference altitude HgtIPP must be determined before this step.)

d. Select the median value of all of the $2 \mathrm{~h}$ slant conversion altitudes as the daily slant conversion altitude (AltIPP).

These processes are supplemented by various plot evaluations. 


\section{The Supplement related to this article is available online at doi:10.5194/angeo-35-599-2017-supplement.}

Competing interests. A. J. Mazzella Jr. is a developer for the SCORPION method. The other authors declare that they have no conflict of interest.

Acknowledgements. Part of E. Yizengaw's work was supported by AFOSR (FA9550-12-1-0437 and FA9550-15-1-0399) and NSF (AGS1450512) grants. Graham J. Bailey provided SUPIM. Development of SCORPION was conducted in collaboration with G. Susan Rao and supported by the Air Force Research Laboratory Space Vehicles Directorate under SBIR contracts FA8718-04-C-0009 and FA8718-05-C-0026 to NorthWest Research Associates. The GPS data were obtained from the Crustal Dynamics Data Information System (CDDIS) at the NASA Goddard Space Flight Center FTP site (ftp://cddis.gsfc.nasa.gov) for the IGS stations (Dow et al., 2009), from the UNAVCO Facility (ftp://data-out.unavco.org/pub/rinex/obs) with support from the National Science Foundation (NSF) and National Aeronautics and Space Administration (NASA) under NSF Cooperative Agreement No. EAR-0735156 for the UNAVCO sites, and from TrigNet (ftp://ftp.trignet.co.za) for Grahamstown. The IONEX data were obtained from the Crustal Dynamics Data Information System (CDDIS) at the NASA Goddard Space Flight Center FTP site (ftp://cddis.gsfc.nasa.gov; Dow et al., 2009), and the associated processing code was obtained from the University of Bern FTP site ftp://ftp.unibe.ch/aiub/ionex/source/ (Schaer et al., 1998). The CODE relative satellite biases and receiver biases were obtained from the University of Bern FTP site ftp://ftp.unibe.ch/aiub/CODE. The ionosonde data were obtained from the Global Ionosphere Radio Observatory (GIRO; http://spase.info/SMWG/Observatory/GIRO; Reinisch and Galkin, 2011), and the analysis results were also obtained from GIRO (http://spase.info/VWO/NumericalData/GIRO/CHARS.PT15M).

The Ap and F10 indices were obtained from the NOAA National Weather Service Space Weather Prediction Center Web site (http://www.swpc.noaa.gov). The Dst indices, for assessment of quiet days, were obtained from the World Data Center for Geomagnetism, Kyoto, Internet site http://wdc.kugi.kyoto-u.ac.jp. The figures were prepared using the Generic Mapping Tools (GMT) graphics (Wessel and Smith, 1998).

Edited by: P. J. Erickson

Reviewed by: two anonymous referees

\section{References}

Andreasen, A. M., Holland, E. A., Fremouw, E. J., Mazzella, A. J., Rao, G. S., and Secan, J. A.: Investigations of the nature and behavior of plasma-density disturbances that may impact GPS and other transionospheric systems, AFRL-VS-TR-2003-1540, Air Force Res. Lab., Hanscom Air Force Base, Mass, 2002.

Andreasen, C. C., Fremouw, E. J., Holland, E. A., Mazzella, A. J., Rao, G.-S., and Secan, J. A.: Further investigations of ionospheric total electron content and scintillation effects on transionospheric radiowave propagation, AFRL-VS-HA-TR-980037, Air Force Res. Lab., Hanscom Air Force Base, Mass, 1998.

Anghel, A., Carrano, C., Komjathy, A., Astilean, A., and Letia, T. Kalman filter-based algorithms for monitoring the ionosphere and plasmasphere with GPS in near-real time, J. Atmos. Sol.Terr. Phy., 71, 158-174, doi:10.1016/j.jastp.2008.10.006, 2009.

Bailey, G. J. and Balan, N.: A low-latitude ionosphereplasmasphere model, in STEP Handbook on Ionospheric Models, edited by: Schunk, R. W., Utah State Univ., Logan, 173-206, 1996.

Belehaki, A., Jakowski, N., and Reinisch, B. W.: Comparison of ionospheric ionization measurements over Athens using ground ionosonde and GPS-derived TEC values, Radio Sci., 38, 1-11, doi:10.1029/2003RS002868, 2003.

Carrano, C. S., Anghel, A., Quinn, R. A., and Groves, K. M.: Kalman filter estimation of plasmaspheric total electron content using GPS, Radio Sci., 44, RS0A10, doi:10.1029/2008RS004070, 2009.

Dow, J. M., Neilan, R. E., and Rizos, C.: The International GNSS Service in a changing landscape of Global Navigation Satellite Systems, J. Geodesy, 83, 191-198, doi:10.1007/s00190-0080300-3, 2009.

Fremouw, E. J., Mazzella, A. J., and Rao, G.-S.: Ionospheric Sensor Developments for the Year-2000 Solar Maximum, AFRL-VSTR-2001-1538, Air Force Res. Lab., Hanscom Air Force Base, Mass, 2001.

Huang, X. and Reinisch, B. W.: Vertical electron content from ionograms in real time, Radio Sci., 36, 335-342, 2001.

Kee, C. and Parkinson, B.: Calibration of multipath errors on GPS pseudorange measurements, paper presented at 7 th International Technical Meeting, Satell. Div., Inst. of Navig., Salt Lake City, Utah, 353-362, Sept. 1994.

Lee, H.-B., Jee, G., Kim, Y. H., and Shim, J. S.: Characteristics of global plasmaspheric TEC in comparison with the ionosphere simultaneously observed by Jason-1 satellite, J. Geophys. Res., 118, 1-12, doi:10.1002/jgra.50130, 2013.

Lunt, N., Kersley, L., and Bailey, G. J.: The influence of the protonosphere on GPS observations: Model simulations, Radio Sci., 34 725-732, doi:10.1029/1999RS900002, 1999a.

Lunt, N., Kersley, L., Bishop, G. J., Mazzella, A. J., and Bailey, G. J.: The effect of the protonosphere on the estimation of GPS total electron content: Validation using model simulations, Radio Sci., 34, 1261-1271, doi:10.1029/1999RS900043, 1999b.

Lunt, N., Kersley, L., Bishop, G. J., and Mazzella, A. J., Jr.: The contribution of the protonosphere to GPS total electron content: Experimental measurements, Radio Sci., 34, 1273-1280, doi:10.1029/1999RS900016, 1999c.

Ma, G. and Maruyama, T.: Derivation of TEC and estimation of instrumental biases from GEONET in Japan, Ann. Geophys., 21, 2083-2093, doi:10.5194/angeo-21-2083-2003, 2003.

Mazzella Jr., A. J.: Plasmasphere effects for GPS TEC measurements in North America, Radio Sci., 44, RS5014, doi:10.1029/2009RS004186, 2009.

Mazzella Jr., A. J.: Determinations of plasmasphere electron content from a latitudinal chain of GPS stations, Radio Sci., 47, RS1013, doi:10.1029/2011RS004769, 2012.

Mazzella, A. J., Holland, E. A., Andreasen, A. M., Andreasen, C. C., Rao, G. S., and Bishop, G. J.: Autonomous estimation of 
plasmasphere content using GPS measurements, Radio Sci., 37, 1092-1095, doi:10.1029/2001RS002520, 2002.

Mazzella, A. J., Rao, G. S., Bailey, G. J., Bishop, G. J., and Tsai, L. C.: GPS determinations of plasmasphere TEC, paper presented at International Beacon Satellite Symposium, Boston Coll., Boston, Mass, 1-7, 2007.

McKinnell, L.-A., Opperman, B., and Cilliers, P. J.: GPS TEC and ionosonde TEC over Grahamstown, South Africa: First comparisons, Adv. Space Res., 39, 816-820, doi:10.1016/j.asr.2006.10.018, 2007.

Reinisch, B. W. and Galkin, I. A.: Global ionospheric radio observatory (GIRO), Earth, Planets, Space, 63, 377-381, doi:10.5047/eps.2011.03.001, 2011.

Schaer, S., Gurtner, W., and Feltens, J.: IONEX: The IONosphere Map EXchange Format Version 1, paper presented at the International GNSS Service Analysis Center Workshop, Darmstadt, Germany, 1-15, 9-11 February 1998.

Secan, J. A., Fremouw, E. J., Mazzella, A. J., Jr., Rasmussen, J., and Snyder, A. L.: Investigation of Plasma Phenomena in the Ionosphere Under Natural Conditions and Under Conditions Artificially Perturbed by HAARP, AFRL-VS-HA-TR-2005-1207, Air Force Res. Lab., Hanscom Air Force Base, Mass, 2005.
Tolman, B., Harris, R. B., Gaussiran, T., Munton, D., Little, J., Mach, R., Nelsen, S., Renfro, B., and Schlossberg, D.: The GPS toolkit-Open source GPS software, paper presented at the 17th International Technical Meeting, Satell. Div., Inst. of Navig., Long Beach, Calif., 2044-2053, September 2004.

Valladares, C. E., Villalobos, J., Hei, M. A., Sheehan, R., Basu, Su., MacKenzie, E., Doherty, P. H., and Rios, V. H.: Simultaneous observation of traveling ionospheric disturbances in the Northern and Southern Hemispheres, Ann. Geophys., 27, 1501-1508, doi:10.5194/angeo-27-1501-2009, 2009.

Wessel, P. and Smith, W. H. F.: New, improved version of Generic Mapping Tools released, Eos Trans. AGU, 79, 579, doi:10.1029/98EO00426, 1998.

Yizengaw, E., Doherty, P., and Fuller-Rowell, T.: Is Space Weather Different Over Africa, and If So, Why? AGU Chapman Conference Report, Space Weather, 11, 389-391, doi:10.1002/swe.20063, 2013.

Yizengaw, E., Moldwin, M. B., Galvan, D., Iijima, B. A., Komjathy, A., and Mannucci, A. J.: Global plasmaspheric TEC and its relative contribution to GPS TEC, J. Atmos. Sol.-Terr. Phy., 70, 1541-1548, doi:10.1016/j.jastp.2008.04.022, 2008. 